Publ. Mat. 56 (2012), 467-471

DOI: 10.5565/PUBLMAT_56212_09

\title{
ON NON-COMMUTING SETS IN FINITE SOLUBLE CC-GROUPS
}

\author{
Adolfo Ballester-Bolinches And John Cossey
}

\begin{abstract}
Lower bounds for the number of elements of the largest non-commuting set of a finite soluble group with a CC-subgroup are considered in this paper.
\end{abstract}

2010 Mathematics Subject Classification: 20D60.

Key words: finite groups, non-commuting sets, CC-subgroups.

A subset of pairwise non-commuting elements in a group $G$ is a subset $T$ of $G$ such that $a b \neq b a$ for all $a, b \in T$. We let $\alpha(G)$ be the order of the largest subset consisting of pairwise non-commuting elements of a finite group $G$. Then $\alpha(G)$ is the size of the maximal empty subgraph of $\Gamma=\Gamma(G)$, the commuting graph associated to $G$. Lower bounds for $\alpha(G)$ have been considered by a number of authors. Bertram [4] found that for a finite group with a CC-subgroup $\alpha(G) \geq|G|^{1 / 3}$. (If $G$ is a group with a proper subgroup $M$ satisfying $C_{G}(x) \leq M$ for each $1 \neq$ $x \in M$ then $M$ is called a CC-subgroup of $G$.) Isaacs found $\alpha(G)$ for extraspecial 2-groups (see [4, p. 40]) and Chin [5] found upper and lower bounds for extraspecial groups of odd order. Some insoluble groups have also been considered. Abdollahi, Akbari and Maimani $[\mathbf{1}]$ found $\alpha(G)$ for $G \cong G L(2, q)$ and Azad and Praeger $[\mathbf{3}]$ found $\alpha(G)$ for $G \cong G L(3, q)$.

Our aim here is to consider finite soluble groups with a CC-subgroup. Bertram [4] shows that $\alpha(G) \geq|G|^{1 / 3}$ for a group with a CC-subgroup. We will show that this bound can be improved for soluble groups by using the classification of finite groups with a CC-subgroup given by Arad and Herfort [2]. They prove that a soluble group with a CC-subgroup is either a Frobenius group or a 2-Frobenius group. We will use the description of Frobenius groups given in [8]. In a Frobenius group the CC-subgroups are the kernel and complements. A group with a normal CC-subgroup $K$ must be a Frobenius group, with $K$ the Frobenius kernel. We say that a group $G$ is a 2-Frobenius group if $G$ has a normal series $K<L<G$ such that $L$ and $G / K$ are Frobenius groups having kernels $K$ and $L / K$, respectively. As an easy consequence of Frobenius group theory one can 
show that $G=K A B, K A=L$ and $A B$ Frobenius groups. Moreover both $A$ and $B$ are cyclic.

To give an estimate of the size of the largest subset of non-commuting elements of a finite soluble group with a CC-subgroup, we use the structure above, but treat Frobenius and 2-Frobenius groups separately. Note that if $G$ is a Frobenius or 2-Frobenius group with Fitting subgroup $F=$ $F(G)$, then the order of $G / F$ is bounded by the square of the order of the smallest chief factor of $G$ in $F$. Since $G$ contains a conjugacy class of non-commuting elements of order $|F|$ it is not surprising that the number of chief factors of $G$ in $F$ will play a role in our estimate.

Theorem 1. Let $G$ be a finite soluble Frobenius group and let $n$ be the number of chief factors of $G$ below $F(G)$ in a chief series of $G$ passing through $F(G)$. Then $\alpha(G) \geq|G|^{n /(n+1)}$.

Proof: Suppose then that $G$ is a Frobenius group with kernel $K$ and complement $H$. If $M<N \leq K$ and $N / M$ is a chief factor of $G$, then $G / M$ is a Frobenius group with kernel $K / M$ and complement $H M / M$. Hence $H$ acts fixed point freely on $N / M$ and so $|H|<|N / M|$. Since the number of conjugates of $H$ in $G$ is $|K|$, and nontrivial elements of distinct conjugates can not commute, we can choose a nontrivial element from each conjugate to form a non-commuting set. Suppose now the number of chief factors of $G$ below $K$ in a chief series of $G$ through $K$ is $n$. We show that $|K|>|G|^{n /(n+1)}$. Consider $|G|^{n}=|K|^{n}|H|^{n}$. Since $|H|$ is less than the order of any chief factor below $K,|H|^{n}<|K|$ and so $|G|^{n}<|K|^{n+1}$.

For the class of Frobenius groups with abelian complement, this lower bound is best possible. We choose a prime $p$ and an integer $n \geq 1$ and then let $K$ be an elementary abelian group of order $p^{n}$. Let $H$ be a cyclic group of order $p-1$ acting as power automorphisms on $K$ and put $G=K H$. Note that $G$ is the disjoint union of the non-trivial elements of the abelian groups $K$ and the conjugates of $H$ and so the size of a maximal non-commuting subset of $G$ is exactly $|K|+1$. We now have

$$
\begin{aligned}
(|K|+1)^{n+1} /|G|^{n} & =\left(p^{n}+1\right)^{n+1} / p^{n^{2}}(p-1)^{n}<\left(p^{n}+1\right)^{n+1} /(p-1)^{n(n+1)} \\
& =\left(\left(1+\frac{1}{p-1}\right)^{n}+\left(\frac{1}{p-1}\right)^{n}\right)^{n+1}
\end{aligned}
$$

and so we can make $\alpha(G)$ as close to $|G|^{n /(n+1)}$ as we want by choosing $p$ large enough. Note in particular when $n=1$ we have $|G|<(|K|+1)^{2}<$ 
$|G|\left(1+\frac{2}{p-1}\right)^{2}$ and so there are examples with $\alpha(G)$ as close to $\sqrt{\mid} G \mid$ as desired.

For 2-Frobenius groups the situation is more complicated. In this case $G$ has Fitting subgroup $F=F(G)$ complemented by a metacyclic group $H=A B$ with $A$ cyclic, normal, of odd order and a core-free CC-subgroup, $B=\langle b\rangle$ a cyclic subgroup acting faithfully on $A$ and $A$ and $B$ of coprime order. Moreover $F B$ is a CC-subgroup. We may not have $|H|$ less than the order of any chief factor of $G$ in $F$ and so the proof above will not hold. We find a slightly worse bound in this case, using similar ideas to the proof of Theorem 1.

We first analyse the action of $H$ on a chief factor of $G$ in $F$. Since $A$ acts fixed point freely on $F$, each chief factor of $G$ below $F$ is faithful as $H$-module. We begin by assuming that $F$ is a minimal normal subgroup of $p$-power order for some prime $p$. We then have $F$ is free as $B$-module and so $F$ is the direct sum of $r$ copies of the regular module by [6, 44.14], [7, B, 5.15], [7, B, 6.21] and [7, B, 5.25]. Thus $|F|=p^{r|B|}$. Let $B=\langle b\rangle$. Suppose that $f b$ and $g b, f, g \in F$, commute. We have $1=[f b, g b]=[f, b]^{b}[b, g]^{b}$ if and only if $1=\left[b, f g^{-1}\right]$. Thus $f b$ and $g b$ commute if and only if $f$ and $g$ are equal modulo the centraliser of $B$ in $F$. Since the regular module contains a unique trivial irreducible submodule, $\left|C_{F}(B)\right|=p^{r}$. Let $\mathcal{S}$ be a transversal for $C_{F}(B)$ in $F$ and set $\mathcal{B}=\{f b: f \in \mathcal{S}\}$. Then $\mathcal{B}$ is a non-commuting subset of $G$. Further if $1 \neq a \in A$ and $\mathcal{B}^{a}=\left\{x^{a}: x \in \mathcal{B}\right\}$, then $\mathcal{B} \cap \mathcal{B}^{a}=\emptyset$ and elements of $\mathcal{B}$ do not commute with elements of $\mathcal{B}^{a}$. If $\mathcal{B}_{1}=\cup_{a \in A} \mathcal{B}^{a}$ then $\mathcal{B}_{1}$ is a non-commuting set containing $|A| p^{r(|B|-1)}$ elements. If $A=\langle a\rangle$ then the set $\left\{a^{f}: f \in F\right\}$ is a non-commuting set and no element of this set commutes with any element of $\mathcal{B}_{1}$. Thus $\alpha(G) \geq|A| p^{r(|B|-1)}+p^{r|B|}=$ $p^{r(|B|-1)}\left(|A|+p^{r}\right)$.

It is now easy to extend this estimate to arbitrary $F$. Since each chief factor is a $|B|^{\text {th }}$ power, we have $|F|=s^{|B|}$ for some $s$ and then $\alpha(G) \geq s^{|B|-1}(|A|+s)$. Note that for small numbers of chief factors, the contribution of elements of order prime to $|A|$ can be significant, but since $s \geq 2^{n}$ if there are $n$ chief factors in $F$ they do not make a significant contribution for large $n$. This is reflected in the proofs below.

Theorem 2. Let $G$ be a finite soluble 2-Frobenius group and let $n$ be the number of chief factors of $G$ below $F(G)$ in a chief series of $G$ passing through $F(G)$, the Fitting subgroup of $G$. Then

(i) if $n \leq 3$ then $\alpha(G) \geq|G|^{2 / 3}$;

(ii) if $n \geq 4$ then $\alpha(G) \geq|G|^{2 n /(2 n+3)}$. 
Proof: We use the notation above.

(i) We prove that $\left(s^{|B|-1}(|A|+s)\right)^{3} \geq|G|^{2}$ or equivalently $\left(s^{|B|-1}(|A|+\right.$ $s))^{3} \geq s^{2|B|}|A|^{2}|B|^{2}$ (note that the proof is independent of $n$ and so this bound holds for all 2-Frobenius groups: (ii) gives better bounds for $n \geq 4$ ). We first dispose of some small values of $|B|$.

If $|B|=2$, then we require $(s(|A|+s))^{3} \geq 4 s^{4}|A|^{2}$ or $|A|^{3}+3|A|^{2} s+$ $3|A| s^{2}+s^{3} \geq 4 s|A|^{2}$. If $s \geq|A|$ then $3|A| s^{2}+s^{3} \geq 4 s|A|^{2}$. If $|A| \geq s$ then $|A|^{3}+3|A|^{2} s \geq 4 s|A|^{2}$. In either case we have the desired inequality.

If $|B|=3$, we require $\left(s^{2}(|A|+s)\right)^{3} \geq 9 s^{6}|A|^{2}$ or $|A|^{3}+3|A|^{2} s+$ $3|A| s^{2}+s^{3} \geq 9|A|^{2}$. Since $|A| \geq 7$ we have $|A|^{3}+3|A|^{2} \geq 10|A|^{2}$, giving the required inequality.

If $|B| \geq 4$, we require $s^{3(|B|-1)}(|A|+s)^{3} \geq s^{2|B|}|A|^{2}|B|^{2}$ or $s^{|B|-3}\left(|A|^{3}+\right.$ $\left.3|A|^{2} s+3|A| s^{2}+s^{3}\right) \geq|A|^{2}|B|^{2}$. It will be enough to show that $s^{|B|-3}\left(|A|^{3}+\right.$ $\left.3|A|^{2} s\right) \geq|A|^{2}|B|^{2}$ or equivalently $s^{|B|-3}(|A|+3 s) \geq|B|^{2}$. Since $|A| \geq$ $|B|$ it will be enough to show $s^{|B|-3} \geq \frac{1}{2}|B|$ and $3 s^{|B|-2} \geq \frac{1}{2}|B|^{2}$. Both inequalities are easily checked. This completes the proof of (i).

(ii) Suppose now that $n>3$. It will be enough to prove that $s^{|B|(2 n+3)} \geq$ $s^{2 n|B|}|A|^{2 n}|B|^{2 n}$ or equivalently $s^{3|B|} \geq|A|^{2 n}|B|^{2 n}$. If $M / N$ is a chief factor of $G$ of smallest order in $F$, then $s^{|B|} \geq|M / N|^{n} \geq|A|^{n}$ and so it will be enough to prove that $s^{|B|} \geq|B|^{2 n}$. Since $s \geq 2^{n}$, it will be enough to show $2^{n|B|} \geq|B|^{2 n}$ or equivalently $2^{|B|} \geq|B|^{2}$. This is easily established unless $|B|=3$ (and is false for $|B|=3$ ). If $|B|=3$, from the observations above either all chief factors in $F$ have order greater than $2^{3}$ or there is at least one with order $2^{3}$. In the first case we have $s \geq 3^{n}$ and then $3^{3 n} \geq 2^{3 n}$. In the second case we must have $|A|=7$. Then it will be enough to show $2^{9 n} \geq 7^{2 n} 3^{2 n}$ and this is immediate, since $2^{9}>(21)^{2}$. This completes the proof of (ii).

Acknowledgement. The first author has been supported by the grant MTM2010-19938-C03-01 from MICINN (Spain).

\section{References}

[1] A. Abdollahi, S. Akbari, and H. R. Maimani, Non-commuting graph of a group, J. Algebra 298(2) (2006), 468-492. DOI: 10.1016/ j.jalgebra.2006.02.015.

[2] Z. Arad And W. Herfort, Classification of finite groups with a CC-subgroup, Comm. Algebra 32(6) (2004), 2087-2098. DOI: 10.1081/AGB-120037209. 
[3] A. Azad And C. E. Praeger, Maximal subsets of pairwise noncommuting elements of three-dimensional general linear groups, Bull. Aust. Math. Soc. 80(1) (2009), 91-104. DOI: 10.1017/S0004972709000057.

[4] E. A. Bertram, Some applications of graph theory to finite groups, Discrete Math. 44(1) (1983), 31-43. DOI: 10.1016/0012-365X(83) 90004-3.

[5] A. Y. M. Chin, On non-commuting sets in an extraspecial p-group, J. Group Theory 8(2) (2005), 189-194. DOI: 10.1515/jgth.2005.8. 2.189.

[6] C. W. Curtis And I. Reiner, "Representation theory of finite groups and associative algebras", Pure and Applied Mathematics XI, Interscience Publishers, a division of John Wiley \& Sons, New YorkLondon, 1962.

[7] K. Doerk and T. Hawkes, "Finite soluble groups", de Gruyter Expositions in Mathematics 4, Walter de Gruyter \& Co., Berlin, 1992.

[8] B. Huppert, Endliche Gruppen. I., Die Grundlehren der Mathematischen Wissenschaften 134, Springer-Verlag, Berlin-New York, 1967.

Adolfo Ballester-Bolinches:

Departament d'Àlgebra

Universitat de València

Dr. Moliner, 50

46100 Burjassot (València)

Spain

E-mail address: Adolfo.Ballester@uv.es

John Cossey:

Department of Mathematics

Mathematical Sciences Institute

Australian National University

Canberra ACT 0200

Australia

E-mail address: John.Cossey@anu.edu.au

Primera versió rebuda el 14 de febrer de 2012, darrera versió rebuda el 15 de març de 2012. 